\section{Dementia

\title{
Effects of Physical Activity in Nursing Home Residents with Dementia: A Randomized Controlled Trial
}

\author{
Marinda Henskens ${ }^{a}$ Ilse M. Nauta ${ }^{b}$ Marieke C.A. van Eekeren ${ }^{c}$ \\ Erik J.A. Scherder ${ }^{d}$ \\ ${ }^{a}$ Department of Clinical Neuropsychology, Vrije Universiteit Amsterdam, Amsterdam, \\ The Netherlands; ${ }^{b}$ Department of Neurology, MS Center Amsterdam, Amsterdam \\ University Medical Center, Amsterdam, The Netherlands; ${ }^{C}$ tanteLouise, Bergen op Zoom, \\ The Netherlands; ${ }^{d}$ Department of Clinical Neuropsychology, Vrije Universiteit Amsterdam, \\ Amsterdam, The Netherlands
}

\section{Keywords}

Dementia · Nursing home - Activities of daily living · Exercise · Cognition · Physical functions · Behavior - Mood

\begin{abstract}
Background/Aims: There is no consensus regarding the optimal nonpharmacological intervention to slow down dementia-related decline. We examined whether physical stimulation interventions were effective in reducing cognitive, physical, mood, and behavioral decline in nursing home residents with dementia. Methods: Eighty-seven nursing home residents with dementia were randomly assigned to 3 physical activity interventions: activities of daily living $(A D L)$ training, multicomponent exercise training, or combined multicomponent exercise and ADL training. Outcomes were measured at baseline, and after 3 and 6 months. Results: A 6-month ADL training benefitted executive functions, physical endurance, and depression among men. Exercise training benefitted only grip strength of participants with mild-to-moderate cognitive impairment. A combined training benefitted functional mobility compared to $A D L$ training, depressive symptoms and agitation compared to exercise training, and physical endurance compared to no physical stimulation. Conclusions: ADL training appears to be effective for nursing home residents with moderately severe dementia. It remains unclear whether exercise training is an effective type of stimulation.


Henskens et al.: Physical Activity in Nursing Home Residents with Dementia

\section{Introduction}

Dementia is characterized by a progressive decline in cognitive and physical functions, and patients often present behavioral problems [1-3]. Cognitive functions affected by dementia typically include memory, global cognition, attention, and executive functions (EF) [4]. EF consist of higher-order cognitive functions, such as planning, set-shifting, taking initiatives, impulse control, and attention [5]. Decline in physical ability is reflected in, among others, loss of mobility, and reduced strength, balance, and walking endurance [6]. Behavioral and psychological symptoms of dementia affect more than $80 \%$ of patients $[1,7-9]$ and include aggression, agitation, apathy, anxiety, and depression [1]. Deterioration in cognitive, physical, and behavioral functions worsens as the disease progresses and is associated with a reduced ability to function independently [10] and a lower quality of life of nursing home $(\mathrm{NH})$ residents with dementia [11].

To date, there is no cure for dementia, and nonpharmacological interventions, such as physical activity, are a promising method for slowing down dementia-related decline [12]. The benefits of physical activity in elderly subjects are well established, including improvements in cognitive functions (in particular EF), balance, strength, walking endurance, mood, and behavioral problems [13-18]. Biological mechanisms, such as alterations in brain structure and function in response to physical activity, are suggested to underlie these benefits $[19,20]$. For instance, physical activity is thought to promote neurogenesis [21] and to increase brain volume and blood flow in the frontal and temporal lobes: brain regions which are vulnerable for dementia-related decline [22]. Stimulating physical activity thus seems to improve brain vitality and may be an appealing intervention to slow down dementiarelated decline $[12,23]$.

Two methods are commonly described in the literature to stimulate physical activity in NH residents with dementia: (1) activities of daily living (ADL) training and (2) exercise training. The first method, ADL training, stimulates physical activity by encouraging residents to perform as much of their self-care as independently as possible during daily care tasks [24]. There is some evidence that ADL training can improve cognitive functions [25], physical functions [24, 26, 27], depressive symptoms [28, 29], and agitation [29]. However, inconsistency remains as other studies do not find benefits of ADL training on these outcomes [26, 30,31]. Possible explanations for the negative results are the small nonrandomized designs [26], or insufficient intensity, frequency (e.g., $15 \mathrm{~min}$ per day/6 days per week) [30], or duration of interventions (majority < 6 months) $[26,27]$. With regard to the second method, exercise training, multiple studies report benefits on physical performance [32-37], cognitive functions [12, 38-44], mood, and behavioral problems [1, 2, 9, 32] in elderly subjects with dementia. Nonetheless, other studies report no benefits of exercise on these outcomes [21, $34,35,43-49]$. The majority of noneffective studies had a short duration ( $<4$ months) [21, 43 , 47] or too low an intensity [44].

Heterogeneity in type, frequency, and duration of physical activity interventions limits the ability to draw firm conclusions about the optimal training for $\mathrm{NH}$ residents with dementia $[21,32,43,47]$. For instance, some studies report the greatest benefits after multicomponent exercise interventions (e.g., combined strength and aerobic exercises) [32, 42], while other studies consider aerobic exercise to be the driving force for improvements in cognition, physical functions, mood, and behavioral problems [1,2,12]. Regarding the duration, there is some evidence that short-term exercise interventions ( $<12$ weeks) are more likely to benefit mood and behavioral problems [9], while other studies report benefits on these outcomes only after 12 weeks of exercise $[36,50]$. Despite the heterogeneity, there is some consensus that the largest improvements in function are found after multicomponent interventions with a duration of at least 12 weeks, with larger effect sizes (ES) found in interven- 
tions with longer durations [36]. In line with this, 6 months of physical activity are found to benefit cognitive and physical functions of $\mathrm{NH}$ residents with dementia [37, 51].

Given that both ADL training and exercise can each, in their own way, positively affect outcomes, a combined exercise and ADL intervention may be a promising method to effectuate further improvements. Therefore, the purpose of this study was to compare the effect of (1) ADL training, (2) multicomponent exercise training (i.e., aerobic and strength), and (3) combined exercise and ADL training on cognitive and physical functions, mood, and behavioral problems of $\mathrm{NH}$ residents with dementia. A 6-month ADL or exercise intervention was hypothesized to maintain or delay the decline in cognitive, physical, mood, and behavioral outcomes compared to a control intervention. A combined exercise and ADL training was expected to produce the greatest benefits.

\section{Methods}

Design

The present study was a double parallel randomized controlled trial with 3 physical activity interventions, which has been described in a previous study [52]. The scientific and Ethical Review Board of the Faculty of Behavior and Movement Sciences of the VU University Amsterdam approved the study (VCWE2015-183R1). Written informed consent was obtained from the participant's legal representatives prior to inclusion.

\section{Randomization}

An independent researcher randomized 11 locations of a Dutch NH to an ADL training or care as usual intervention. Subsequently, participants at each location were individually assigned to an exercise or control intervention using a random number generator with random block sizes and a random number of blocks. Recruitment procedures have been described in a previous study [52]. In total, 4 groups were compared: (1) ADL: ADL training, (2) EX: exercise training, (3) EX-ADL: combined exercise and ADL training, and (4) CO: no physical activity intervention (Fig. 1).

Sample Size

A power calculation was performed based on the Erlangen-ADL [53], the primary outcome measure of the trial, which we have described in a previous study [52]. With an $\alpha$-value set at $0.05, \beta$ at 0.80 , an intraclass correlation of 0.6 within the participant, and an intraclass correlation of 0.1 within the location, we required a group of 120 participants to detect a difference of 0.48 standard deviations (SD) in the outcome variable with a comparison between 2 groups. With a comparison between 4 groups, we can detect a difference of 0.68 SD in outcome.

\section{Participants}

Inclusion criteria were (1) living on a psychogeriatric ward of NH "tanteLouise," (2) diagnosis of dementia, (3) functional mobility level A or B (according to the Arjo mobility gallery; residents with level A are ambulatory, but may use a walking stick; residents with level B use a walking aid) [54], (4) age $\geq 65$ years, (5) a score on the Mini-Mental State Examination (MMSE) between 1 and 24 [55], and (6) able to complete the 6-min walking test (6MWT) (with or without walking aid) [56]. Exclusion criteria were (1) wheelchair bound [54], (2) very poor vision, (3) severe cardiovascular problems, (4) instable diabetes mellitus, or (5) aggressive or runaway behavior.

\section{Demographics}

Factors considered were age, gender, educational level, type and severity of dementia, and comorbidities. Participants were classified as having received low (score 1-3), medium (score 4-5), or high (score 6-7) education using the classification of Verhage [57]. Severity of dementia was determined using the Global Deterioration Scale [58], with scores ranging from 1 (no cognitive decline) to 7 (very severe). Type of dementia and comorbidities were derived from the patients' medical charts. Comorbidities were categorized according to the Dutch translation of the Long-Term Care Facility Resident Assessment Instrument, section 


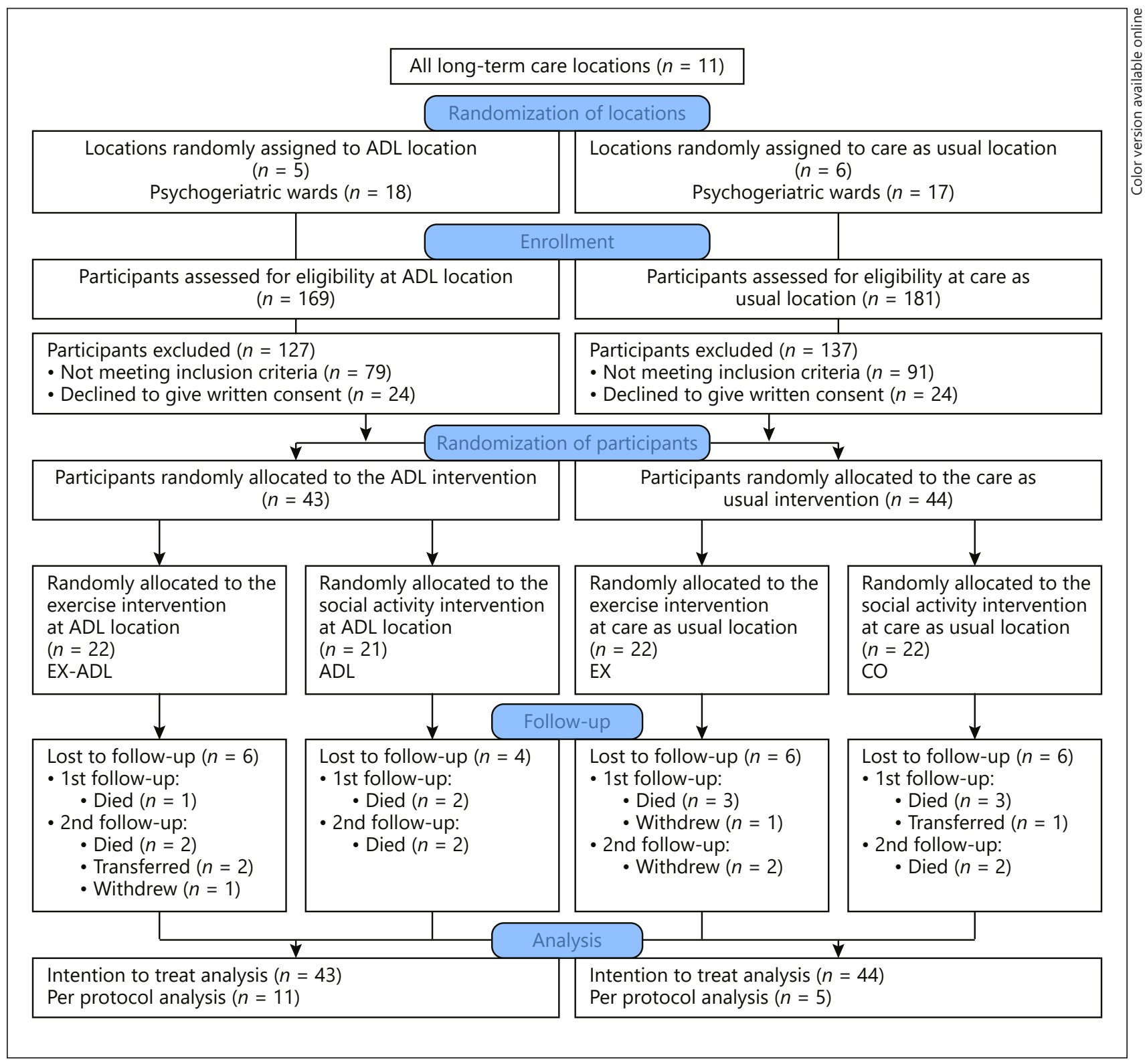

Fig. 1. Flowchart of the sampling procedure. EX-ADL, exercise and ADL; ADL, social activity and ADL; EX, exercise and control; CO, social activity and control.

I disease diagnosis [59]. Comorbidities were reported in the following categories: endocrine/metabolic/ nutritional, heart/blood circulation, musculoskeletal, neurological, sensory, psychiatric/mood, pulmonary, and other. The sum of the subcategories was used as a total comorbidity score.

\section{Materials and Procedure}

Materials

This study is part of a larger trial examining the effects of physical activity interventions in NH residents with dementia. The primary outcome measure of the trial is ADL ability, which we have described in a previous article [52]. This study examines the secondary outcome measures of the trial, which include cognitive functions, physical functions, mood, and behavior. The outcome measures were assessed at baseline, and after 3 and 6 months, except for the MMSE [55], which was measured at baseline and after 6 
Henskens et al.: Physical Activity in Nursing Home Residents with Dementia

months. The cognitive outcomes were administered by trained neuropsychology students, and the physical outcomes by human movement science students, who were all blinded to group allocation. The depression, apathy, and agitation questionnaires were completed by nursing staff who, due to the nature of the intervention, could not be blinded to group allocation.

Cognitive Function

Cognitive impairment was measured with the MMSE (scored from 0 to 30; scores below 24 indicate cognitive impairment) [55] and the Severe Impairment Battery - Short Form (SIB-S, scored from 0 to 50; higher scores indicate better cognitive functions) [60]. The MMSE and SIB-S are reliable and valid tools for elderly subjects with dementia [61]. EF was measured using the category fluency subtest of the Groninger Intelligentie Test [62, 63], the Wechsler Digit Span Task Backward (scored from 0 to 21) [64], and the go-no-go test and conflicting instructions test of the Frontal Assessment Battery (scored from 0 to 3) [65]. An "EF" domain was created which is the average of the sum of the z-scores of the category fluency task, go-no-go test, conflicting instructions test, and the digit span task backward. Attention was measured with the Digit Span Task Forward (DSF, scored from 0 to 21) [64]. Higher EF and DSF scores indicate better functioning. All EF instruments and the DSF were administered only to participants with an MMSE of 5 or higher.

Physical Function

Physical Endurance. The 6MWT measured physical endurance [56]. Participants were asked to walk the longest distance possible within $6 \mathrm{~min}$. The outcome was total walking distance in meters, with higher scores indicating better performance.

Functional Mobility. The timed up and go test measured functional mobility [66]. The outcome measure was time, in seconds, taken to stand up from a chair, walk $3 \mathrm{~m}$, turn, return to the chair, and sit down. Lower scores indicate better performance.

Balance. The figure-of-8 walk test [67] and the Frailty and Injuries: Cooperative Studies of Intervention Techniques-4 scale (FICSIT-4) measured dynamic balance and static balance, respectively [68]. A "balance" domain was created which is the average of the sum of the z-scores of the figure-of- 8 walk test and the FICSIT-4, with higher scores representing better balance.

Muscle Strength. The timed chair stand test 5 repetitions measured lower limb strength [69]. The outcome was time, taken in seconds, to stand up from a seated position 5 times. Lower scores indicate better performance. The Jamar hand dynamometer assessed hand grip strength bilaterally [70]. The physical performance tests used in this study are reliable for use among elderly subjects with dementia [71-73].

Mood and Behavior

Depressive symptoms were measured using the Cornell Scale for Depression in Dementia (scored from 0 to 38; higher scores indicate more depressive symptoms) [74]. Apathy was measured using the Apathy Evaluation Scale-10 (scored from 10 to 40; higher scores indicate more apathetic behavior) [75]. The CohenMansfield Agitation Inventory (CMAI) measured the frequency of agitated behaviors [76, 77]. Symptoms can be clustered into aggressive behavior (scored from 9 to 63), physically nonaggressive behavior (scored from 6 to 42), and verbally agitated behavior (scored from 5 to 35). The depression, apathy, and agitation questionnaires are sufficiently reliable for use in $\mathrm{NH}$ residents with dementia $[75,78,79]$.

\section{Procedure}

Interventions

ADL Training. The ADL intervention is an individually based training for participants, in which nursing staff stimulated participants to perform as much of their daily self-care and activities as independently as possible throughout the day. This includes areas of self-care (e.g., getting dressed, bathing, and eating) as well as household activities (e.g., making the bed, setting the table, and doing the dishes). Participants performed these tasks either with or without verbal instructions from the staff. For each participant, individually based goals, plans, capabilities, and activities were reported in the following 4 domains of the health care plan: (1) living conditions, (2) participation, (3) mental well-being and autonomy, and (4) physical well-being and health. The person-centered approach allowed for variance in training based on someone's capabilities and limitations. In a previous study, we have provided a detailed example of an individually based health care plan [52]. Prior to starting the ADL intervention, a group of "ambassadors" (consisting of 1 physio- or occupational therapist and 2 nursing staff) received a 3-h educational session. In this session, qualified physio- 
Henskens et al.: Physical Activity in Nursing Home Residents with Dementia

and occupational therapists provided ambassadors with information about the benefits of physical activity, and how to (safely) stimulate residents to perform daily care and activities independently. Two additional 3 -h educational sessions were provided 6 and 14 weeks into the intervention, in which ambassadors also had the opportunity to ask questions and receive feedback from the trainers about problems which they (or other staff members) may have encountered during the ADL intervention. Ambassadors were responsible for sharing their knowledge about ADL stimulation with other staff members and warranting the continuation of ADL training at their ward. Participants in the control locations received care as usual.

Exercise Training. Qualified movement teachers guided the multicomponent exercise training, which consisted of alternating strength and aerobic sessions. All sessions were group-based (4-6 residents) and took place 3 times per week for 6 months. Sessions were alternated weekly (e.g., a week of 2 strength and 1 aerobic session, followed by a week of 1 strength and 2 aerobic sessions). Each session lasted 30-45 min, with a progressive increase in intensity. Strength exercises were performed from a seated position and concentrated on upper extremities (i.e., chest press, side and front raises, triceps/biceps curl, seated row), lower extremities (i.e., squats, calf raises, seated leg extension, standing rear leg raise, hip abduction), and the torso (i.e., chair crunch, seated back extension). Exercises were intended to start with 3 sets of 8 repetitions, which could be increased to 10,12, or 15 repetitions, and subsequently with additional weights, only after correct execution. The aerobic training consisted of outdoor walking sessions. Participants were assigned to either a short walking route $(500 \mathrm{~m})$ or a long route $(1 \mathrm{~km})$, depending on their performance on the 6MWT. In a group of 4-6 participants, 1 movement therapist guided 2 participants to guarantee individual attention and safety, to optimally match participants on their walking pace and to minimize differences in fitness level within a group. The intensity of the training was increased by increasing either the walking distance or the walking speed by means of interval training. Participants in the control group drank tea with the nursing staff 3 times per week to control for the social aspect of the exercise intervention.

\section{Compliance to the Intervention}

To determine compliance to the ADL intervention, 23 ambassadors from the ADL locations were asked to complete a monthly online questionnaire. The questionnaire consisted of 3 questions pertaining to the extent to which ambassadors: (1) stimulated residents to perform more ADL activities independently, (2) motivated other staff members to stimulate resident independence, and (3) took over care tasks that residents were able to perform independently. All questions were rated on a scale from 0 to 100 . To determine compliance to the exercise intervention, movement teachers reported participants' attendance (or reasons for absence) to the exercise sessions, as well as the intensity of strength and aerobic sessions (i.e., number of sets and repetitions, and distance walked, respectively).

\section{Statistical Analysis}

Data were analyzed using IBM SPSS 21.0. One-way independent ANOVAs and Pearson's $\chi^{2}$ tests were used to compare groups at baseline. To evaluate the effects of the interventions, multilevel analyses were performed using a 3-level model: (1) observations, (2) participants, and (3) NH. A modified intention-to-treat approach was used: we included all participants with at least 1 post-baseline assessment. Two models were used to analyze the data: (1) overall intervention effects, independent of time, and (2) differences in intervention effects over time. Regression coefficients were reported, representing an overall intervention effect for model 1 (group estimate), and the difference in intervention effect at different time points for model 2 (group-by-time estimate). No imputation method was used for missing data, as multilevel analysis is flexible in handling missing data $[80,81]$. To evaluate the effect of the ADL training, the ADL group (EX-ADL and ADL) was compared to the group receiving no ADL training (EX and CO). To examine the effect of the exercise training, the exercise group (EX-ADL and EX) was compared to the group receiving no exercise training (ADL and $\mathrm{CO}$ ). To evaluate the effect of the combined exercise and ADL intervention, EX-ADL was compared to the "ADL only" group (ADL), the "exercise only" group (EX), and the group receiving none of the two physical activity interventions (CO). All analyses were adjusted for baseline performance, age, and gender. Analyses were stratified for gender or MMSE if these were effect modifiers, in which case results were reported separately for groups. Statistical significance was determined at $p<0.05$. A Bonferroni correction was used to correct for $\alpha$-inflation for the "EF" domain, EF tasks, and the DSF ( $p<0.01[0.05 / 5])$, the "balance" domain $(p<0.017$ [0.05/3]), and the subscales and overall score of the CMAI $(p<0.017$ [0.05/3]). ES (Cohen's $d$ ) were calculated by dividing the regression coefficient by the standard deviation of the outcome variable. Cohen's $d$ of $0.2,0.5$, and 0.8 were considered small, moderate, and large ES, respectively [82]. 
Table 1. General baseline characteristics of nursing home residents with dementia $(n=87)$

\begin{tabular}{|c|c|c|c|c|}
\hline & $\operatorname{EX}-\operatorname{ADL}(n=22)$ & $\operatorname{ADL}(n=21)$ & $\operatorname{EX}(n=22)$ & $\mathrm{CO}(n=22)$ \\
\hline Age (mean $\pm S D)$, years & $86.95 \pm 7.21$ & $86.05 \pm 5.86$ & $85.14 \pm 4.64$ & $84.73 \pm 4.55$ \\
\hline Age (range), years & $75-100$ & 71-95 & 75-93 & 75-94 \\
\hline Gender (female), $n(\%)$ & $14(63.4)$ & $19(90.5)$ & $17(77.3)$ & $17(77.3)$ \\
\hline GDS (mean \pm SD) & $5.05 \pm 0.80$ & $5.19 \pm 0.84$ & $5.40 \pm 0.82$ & $5.55 \pm 0.83$ \\
\hline GDS (range) & $3-6$ & $4-6$ & $3-6$ & $3-6$ \\
\hline Total comorbidities (mean \pm SD), $n$ & $4.64 \pm 2.04$ & $4.19 \pm 1.86$ & $4.45 \pm 1.71$ & $4.57 \pm 2.04$ \\
\hline Endocrine/metabolic/nutritional, $n(\%)$ & $10(45.5)$ & $3(14.3)$ & $9(40.9)$ & $6(28.6)$ \\
\hline Heart/blood circulation, $n(\%)$ & $17(77.3)$ & $15(71.4)$ & $18(81.8)$ & $18(85.7)$ \\
\hline Musculoskeletal, $n(\%)$ & $15(68.2)$ & $12(57.1)$ & $14(63.6)$ & $12(57.1)$ \\
\hline Neurological, $n(\%)$ & $7(31.8)$ & $9(42.9)$ & $8(36.4)$ & $9(42.9)$ \\
\hline Sensory, $n(\%)$ & $8(36.4)$ & $7(33.3)$ & $10(45.5)$ & $10(47.6)$ \\
\hline Psychiatric/mood, $n(\%)$ & $2(9.1)$ & $2(9.5)$ & $2(9.1)$ & $4(19)$ \\
\hline Pulmonary, $n(\%)$ & $2(9.1)$ & $4(19)$ & $2(9.1)$ & $2(9.5)$ \\
\hline Other, $n(\%)$ & $12(54.5)$ & $10(47.6)$ & $13(61.9)$ & $12(57.1)$ \\
\hline \multicolumn{5}{|l|}{ Education, $n(\%)$} \\
\hline Low (1-3) & $8(36.4)$ & $9(42.9)$ & $8(36.4)$ & $3(13.6)$ \\
\hline Medium (4-5) & $12(54.5)$ & $9(42.9)$ & $10(45.5)$ & $11(50)$ \\
\hline High (6-7) & $2(9.1)$ & $2(9.5)$ & $1(4.5)$ & $3(13.6)$ \\
\hline \multicolumn{5}{|l|}{ Diagnosis, $n(\%)$} \\
\hline $\mathrm{AD}$ & $6(27.3)$ & $6(28.6)$ & $14(63.6)$ & $8(36.4)$ \\
\hline $\mathrm{VaD}$ & $3(13.6)$ & $5(23.8)$ & $1(4.5)$ & $3(13.6)$ \\
\hline Mixed VaD and AD & $3(13.6)$ & $1(4.8)$ & $1(4.5)$ & $1(4.5)$ \\
\hline Other/unknown & $10(45.5)$ & $9(42.9)$ & $6(27.3)$ & $9(40.9)$ \\
\hline
\end{tabular}

GDS, Global Deterioration Scale; EX-ADL, exercise and ADL; ADL, social activity and ADL; EX, exercise and control; CO, social activity and control; AD, Alzheimer's disease; VaD, vascular dementia.

\section{Results}

\section{Sample}

In total, there were 350 residents living at the psychogeriatric wards of the $\mathrm{NH}$. All residents were assessed for eligibility. We were able to include 87 participants from the intended 120 participants (Fig. 1). Baseline demographic characteristics for the randomized groups were well matched (Table 1). The average MMSE scores are indicative of a sample with a moderately severe stage of dementia [83]. In total, 10 participants were excluded from the EF analyses due to an MMSE score below 5. Most baseline scores on outcome measures did not differ significantly between groups, except for the 6MWT and grip strength (Table 2). The dropout rate was not significantly different among the 4 groups (EX-ADL 24\%, ADL 23\%, EX $27 \%$, CO $27 \%,=0.57, p=0.89$ ). Participants who dropped out were slightly older (88 vs. 85 ) and had a higher Global Deterioration Scale score (5.63 vs. 5.19).

\section{Compliance to the Intervention}

ADLIntervention. The response rate to the monthly questionnaires to measure compliance to the ADL intervention was $76 \%$. Mean compliance to the intervention was $68 \%$, which includes the following 3 aspects: (1) $69 \%$ of the time, nursing staff succeeded in letting residents perform care tasks independently, (2) $66 \%$ of the time, staff managed to stimulate the participants to perform more ADL activities independently, (3) 70\% of the time, ambassadors succeeded in motivating other staff members to stimulate residents. The main reasons for not succeeding to stimulate residents were fatigue (54\%) or unwillingness (45\%) of the resident. 
Dementia

Cognitive Disorders
Dement Geriatr Cogn Disord 2018;46:60-80

DOI: 10.1159/000491818

(c) 2018 The Author(s). Published by S. Karger AG, Basel www.karger.com/dem

Henskens et al.: Physical Activity in Nursing Home Residents with Dementia

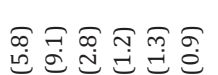

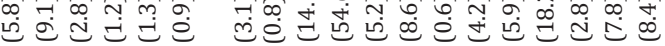

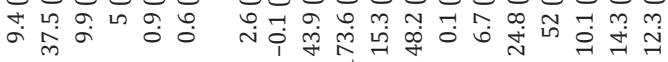

\section{กิ \\ 0 过过包}

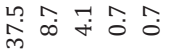

ตำ

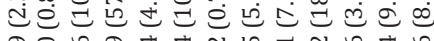

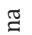

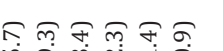

-

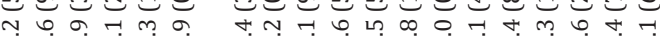

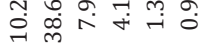

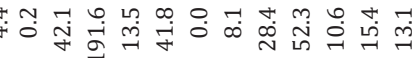

กูก

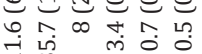

กำ จำ

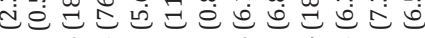

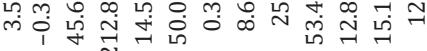

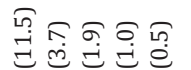

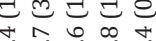
๘

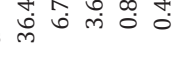

m็

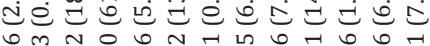

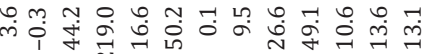

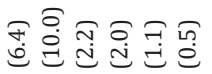

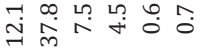

อே สํำ

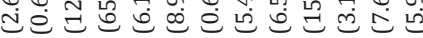

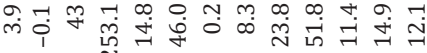

접요

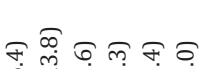

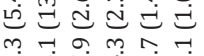

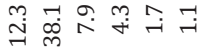

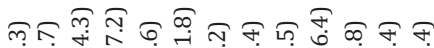

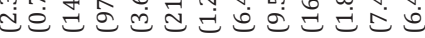

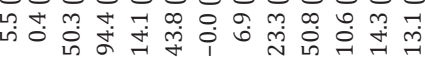

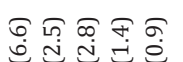

0 in $10-1$ ஐ

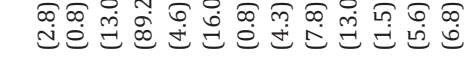

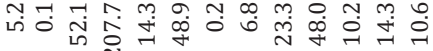

ㄷํำ ำ

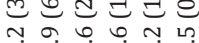

รอำ

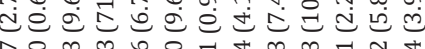

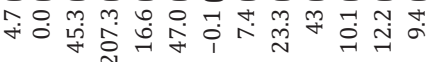

mit

กู

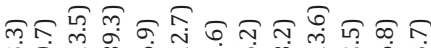

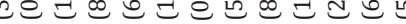

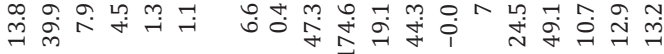

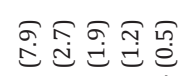

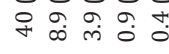

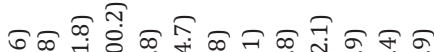

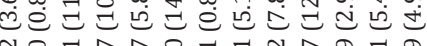

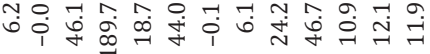

อ ம்

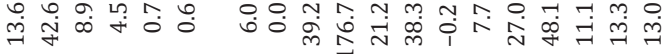

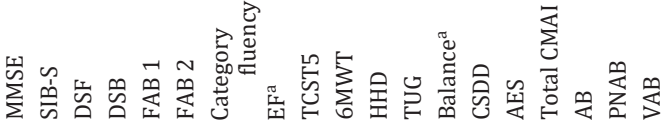

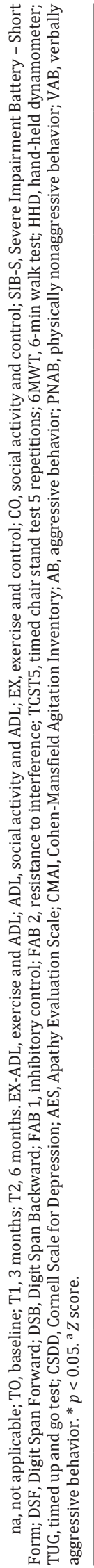




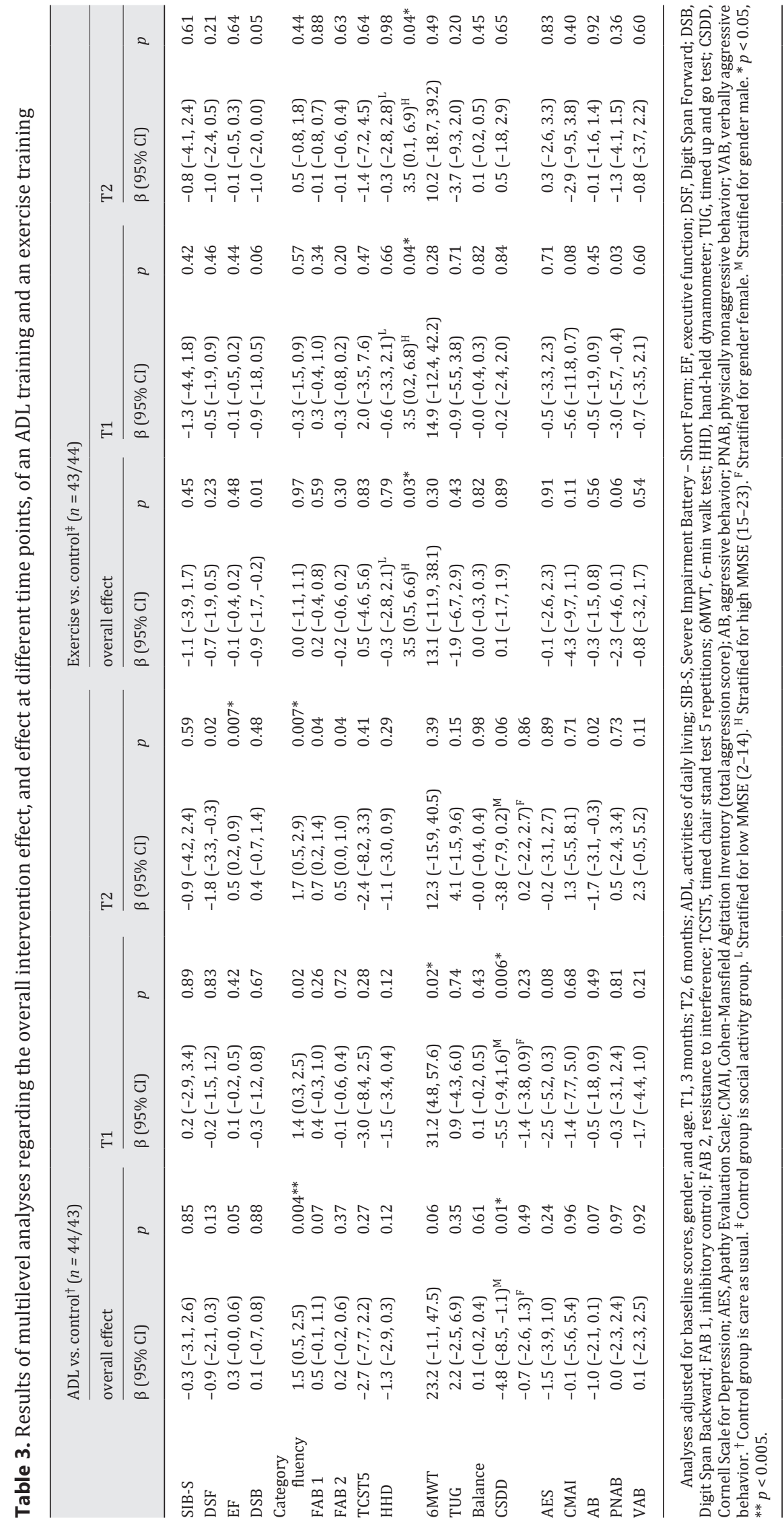


Dementia

Cognitive Disorders
Dement Geriatr Cogn Disord 2018;46:60-80

\begin{tabular}{l|l|l}
\hline DOI: $10.1159 / 000491818$ & (c) 2018 The Author(s). Published by S. Karger AG, Basel \\
\hline
\end{tabular} www.karger.com/dem

Henskens et al.: Physical Activity in Nursing Home Residents with Dementia

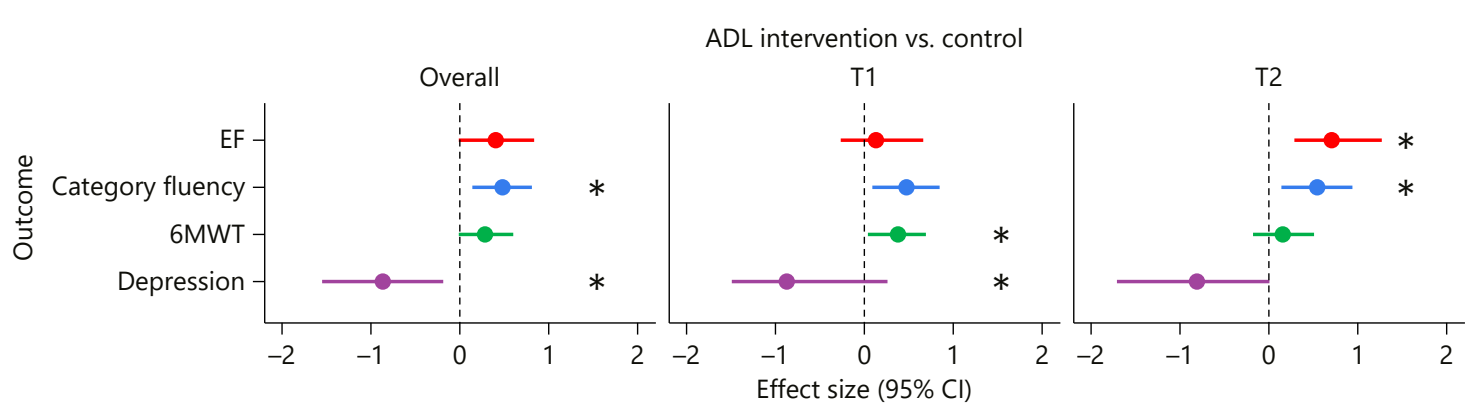

Fig. 2. Forest plot of overall effect sizes and effect sizes after 3 (T1) and 6 months (T2) of ADL training. Effect sizes represented in Cohen's $d$; all outcomes are in favor of the ADL group. CI, confidence interval; ADL, activities of daily living; EF, executive functions; 6MWT, 6-min walk test. ${ }^{*} p<0.02$ : significant differences in outcome measure between groups.

Staff also indicated that it was sometimes faster to take over care tasks, which felt necessary due to high workload, but also because residents did not understand certain tasks.

Exercise. Mean attendance to the intended 72 exercise sessions was 55\% (mean $=39.5$, $\mathrm{SD}=20.8$; range $=0-64$ ). There was no significant difference in attendance between the aerobic and strength sessions. The main reasons for absence were influenza, norovirus, lack of motivation, overlapping activities, and bank holidays. The intensity of the training was not always consistently reported. However, the intensity reports that were available suggest that there were a few deviations from the intended exercise protocol: mainly 2 sets of repetitions were trained instead of the intended 3 , and participants did not always achieve the $500 \mathrm{~m}$ or $1 \mathrm{~km}$ walking distance due to time constraints.

\section{Intention-to-Treat Analysis}

Adjusted multilevel models providing overall intervention effects and intervention effects after 3 and 6 months of the ADL training and the exercise intervention are presented in Table 3.

Effect of ADL Training

Cognitive Functions. There was no significant main effect of the ADL training on the MMSE $(b=0.18, t(54)=0.22, p=0.83)$, the SIB-S, DSF, or the EF domain when compared to the control group (Table 3). A significant group-by-time interaction was found for the EF domain, as the ADL group performed better than the group receiving no ADL training on EF after 6 months of training ( $p=0.007$; Table 3 ). The Cohen's $d$ ES was 0.69 , indicating a medium ES (Fig. 2). When examining the separate EF tasks, a significant main effect of ADL training was found on the category fluency task $(p=0.004)$, showing a small-to-medium ES (Cohen's $d=$ 0.48 ; Fig. 2). This indicates that on average over time, ADL training had a positive effect on category fluency when compared to no ADL training. The largest difference in category fluency performance between the ADL group and the control group was observed after 6 months of training ( $p=0.007$ ), with a medium ES (Cohen's $d=0.54$ ).

Physical Functions. One significant group-by-time interaction was found of the ADL training on the 6MWT, which indicates that after 3 months, the ADL group was able to walk longer distances than the group who received no ADL training $(p=0.02)$, showing a small-tomedium ES (Cohen's $d=0.38$ ). Group differences in favor of the ADL group were still present 


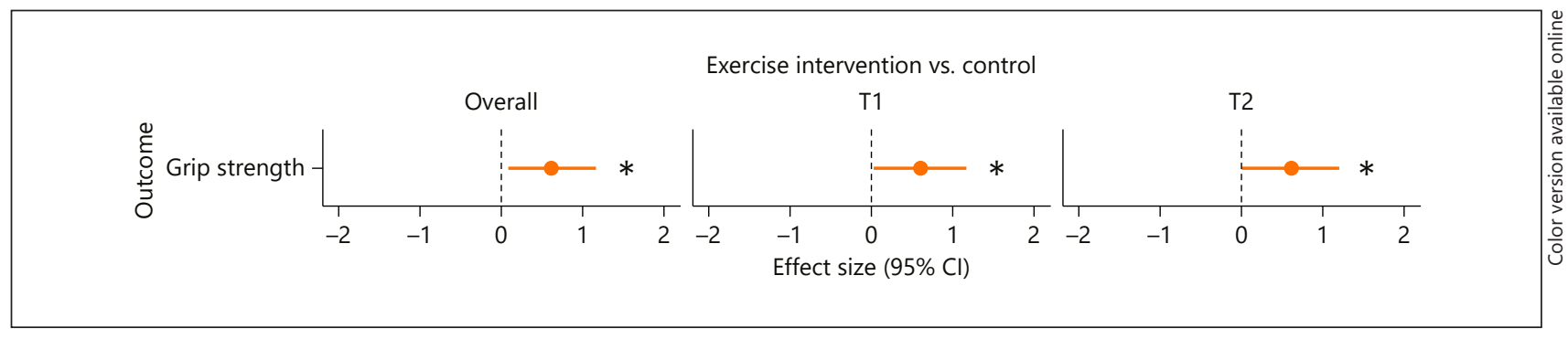

Fig. 3. Forest plot of overall effect size and effect sizes after 3 (T1) and 6 months (T2) of exercise training. Effect sizes represented in Cohen's $d$; outcome is in favor of the exercise group. CI, confidence interval. ${ }^{*} p<$ 0.05: significant differences in outcome measure between groups.

after 6 months, but no longer significant. There were no effects of ADL training on other physical functions (Table 3).

Mood. There was a significant main effect of ADL training on depressive symptoms when analyses were stratified for gender. This result indicates that, on average over time, ADL training had a positive effect on depressive symptoms among men $(p=0.01)$, with a large ES (Cohen's $d=0.86$ ). There was no main effect of ADL training among women. In addition, a significant group-by-time interaction was found for depressive symptoms after 3 months among men, which indicates that after 3 months, men in the ADL group had fewer depressive symptoms compared to men who received no ADL training ( $p=0.006$; Cohen's $d=0.87$ ). Group differences in depressive symptoms in favor of the ADL group were still present, but no longer significant, after 6 months $(p=0.06)$. No significant effect of ADL training was found on apathy.

Behavior. After correcting for $\alpha$-inflation, no significant effects of ADL training were found for the 3 subscales and the total score of the CMAI when compared to no ADL training.

\section{Effect of Exercise Training}

Cognitive Functions. After correcting for $\alpha$-inflation, no significant effects were found of the exercise training on the MMSE $(b=0.19, t(54)=0.23, p=0.82)$ or other cognitive functions when compared to no exercise (Table 3).

Physical Functions. There was a significant main effect of exercise on grip strength, when analyses were stratified for MMSE. This indicates that, on average over time, exercise had a positive effect on grip strength among participants with mild-to-moderate cognitive impairment (MMSE score 15-23) compared to no exercise ( $p=0.03)$. The Cohen's $d=0.61$, indicating a medium ES (Fig. 3). In addition, significant group-by-time interactions with medium ES were found for grip strength when analyses were stratified for MMSE. Specifically, participants with mild-to-moderate cognitive impairment in the exercise group performed better on grip strength after 3 months ( $p=0.04$, Cohen's $d=0.60)$ and after 6 months ( $p=0.04$; Cohen's $d=0.60)$ compared to participants who received no exercise training. There was no significant effect of exercise on grip strength for participants with moderately severe-to-severe cognitive impairment (MMSE score 2-14). There was no effect of exercise on other physical functions.

Mood. No significant effect of exercise was found for depressive symptoms or apathy when compared to no exercise.

Behavior. After correcting for $\alpha$-inflation, no significant effect of exercise was found for the 3 subscales and the total score of the CMAI when compared to no exercise. 


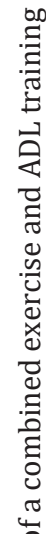

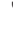

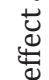

氞

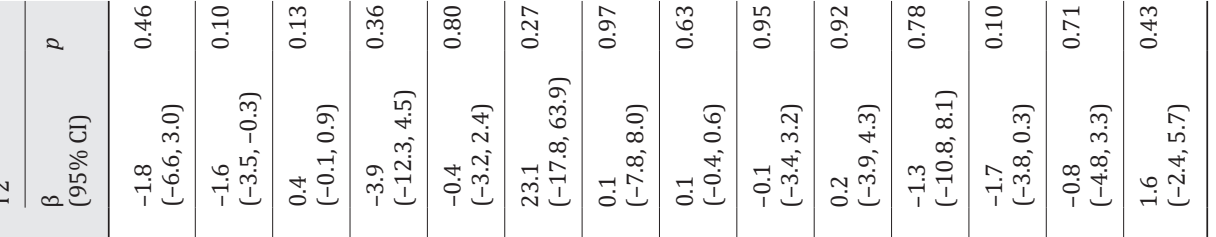

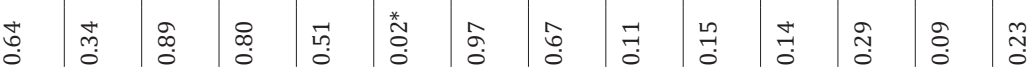




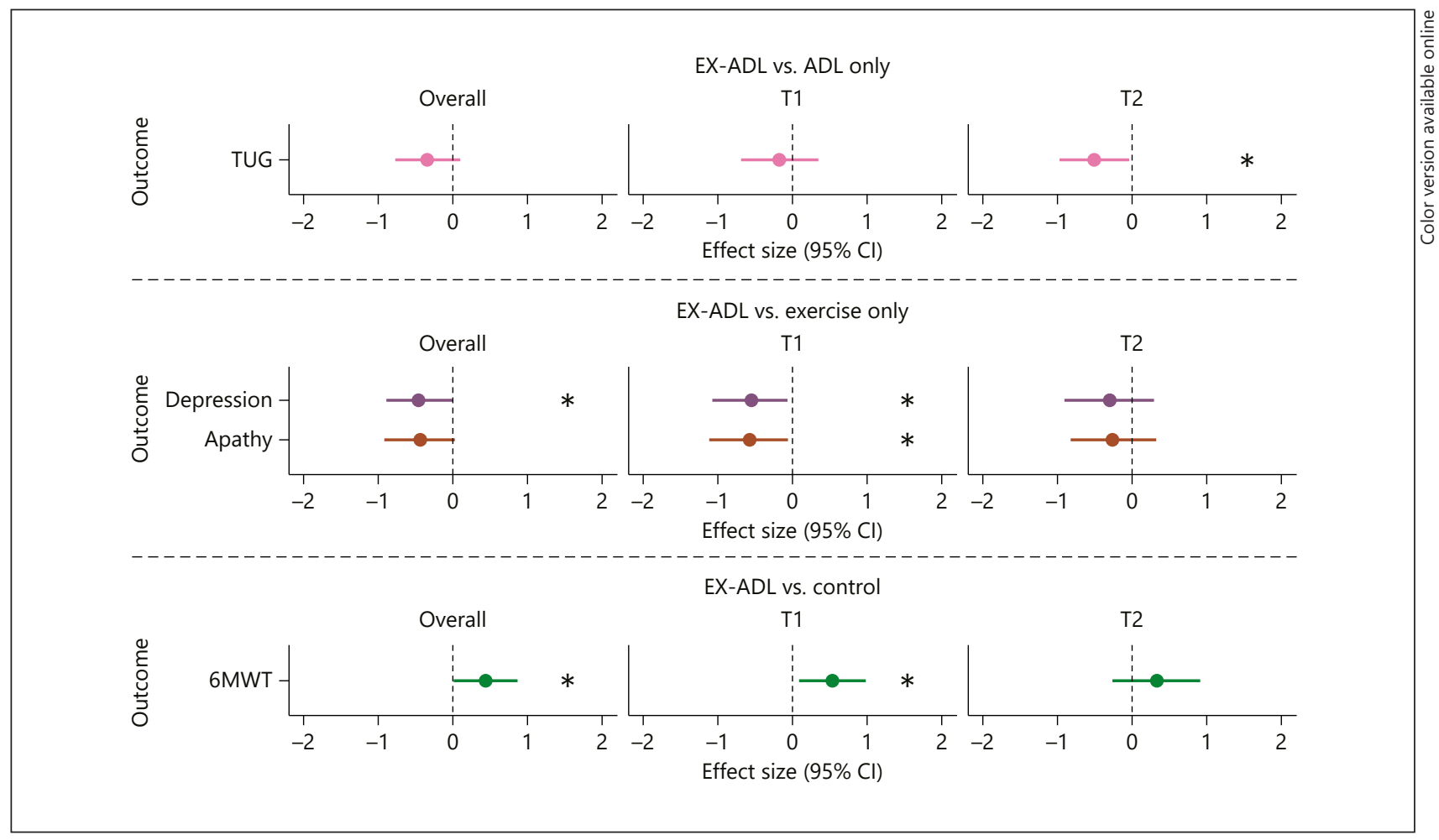

Fig. 4. Forest plot of overall effect sizes and effect sizes after 3 (T1) and 6 months (T2) of a combined exercise and ADL training. Effect sizes represented in Cohen's $d$; all outcomes are in favor of the EX-ADL group. $\mathrm{CI}$, confidence interval; EX-ADL, exercise and ADL training; ADL, activities of daily living; TUG, timed up and go test; 6MWT, 6-min walk test. * $p<0.05$ : significant differences in outcome measure between groups.

Effect of a Combined Exercise and ADL Training

Table 4 presents overall intervention effects, as well as intervention effects after 3 and 6 months of the combined exercise and ADL training (EX-ADL) compared to (1) ADL training, (2) exercise training, and (3) no physical activity stimulation (CO).

$E X-A D L$ versus $A D L$. There was a significant group-by-time effect of the combined exercise and ADL training on the timed up and go test, which indicates that after 6 months, participants who received both interventions performed the timed up and go test faster than participants who only received the ADL training $(p=0.04)$. The Cohen's $d=0.50$, indicating a medium ES (Fig. 4). No positive effects were found of the combined training on other physical functions, cognitive functions, mood, or behavior when compared to the "ADL only" training (Table 4).

$E X-A D L$ versus Exercise. After correcting for $\alpha$-inflation, no significant main effects were found of the combined training on cognitive functions when compared to the "exercise only" training (Table 4). A significant main effect was found of the combined training on depressive symptoms, indicating that on average over time, participants who received the combined training had significantly less depressive symptoms $(p=0.04)$ compared to participants who received only exercise training. The ES was small to medium (Cohen's $d=0.44$ ). In addition, after 3 months, participants who received the combined training had significantly fewer depressive symptoms ( $p=0.03$; Cohen's $d=0.54)$ and lower apathy scores $(p=0.03$; Cohen's $d=0.57$ ) compared to participants who received only exercise training. After 6 months, these differences were no longer significant. There was no significant effect of the combined 
training on physical functions or the CMAI when compared to the "exercise only" intervention (Table 4).

EX-ADL versus No Physical Activity. Both the participants in the combined group and the participants who received no intervention declined in walking distance over time (Table 2). However, there was a main effect of the combined training on the 6MWT, which indicates that on average over time, the participants who received the combined training were able to walk significantly longer distances than the participants who received no intervention $(p=0.04)$. The ES was small to medium (Cohen's $d=0.44$ ). In addition, there was a significant group-bytime interaction effect for the 6MWT, indicating that after 3 months, participants who received the combined training performed better on the 6MWT than participants who received no intervention ( $p=0.02$ ). The Cohen's $d=0.53$, indicating a medium ES. This difference was still present after 6 months, but no longer significant. After adjusting for $\alpha$-inflation, there was no positive effect of the combined training on other physical functions, cognitive functions, mood, or behavior when compared to no physical activity intervention (Table 4).

\section{Discussion}

The present study evaluated the effect of 3 physical activity interventions (i.e., ADL training, exercise training, and a combined exercise and ADL training) on cognitive functions, physical functions, mood, and behavior among $\mathrm{NH}$ residents with dementia. Our results suggest that a 6-month ADL training positively affects cognitive, physical, and mood outcomes. With regard to cognition, positive effects were found for EF, which became apparent after 6 months, and were reflected in improvements in verbal category fluency. Our results are consistent with a previous 3-month ADL training who also found benefits on EF. The authors hypothesized that these benefits from ADL training on EF may result from the appeal which ADL training makes on skills reliant on procedural memory (i.e., the memory necessary for knowing how to perform tasks without the need for conscious thought or attention) [25]. In order to complete basic tasks, cognitive or motor skills are involved which are retrieved automatically from the procedural memory. In elderly subjects with dementia, attentional processes involved in performing ADLs may be re-automatized by training procedural memory so that a task becomes an automatic process instead of a conscious task [25]. Attentional processes can then be disengaged and be used in other tasks, such as semantic fluency. In line with this hypothesis, it has been suggested that problems on the verbal category fluency task are related to problems with attentional capacity [84]. Other studies have also discussed the association between EF and ADL abilities, assuming a relationship between everyday tasks and underlying cognitive mechanisms [85]. Improved performance on the category fluency task is an important indicator of independent functioning, as it relates to daily production tasks (e.g., making a shopping list) and enables participants to link related concepts [86]. EF constitute one of the most important cognitive functions involved in maintaining ADL performance and independent functioning [85]. Therefore, the finding that ADL training improves $\mathrm{EF}$ in $\mathrm{NH}$ residents with dementia is clinically relevant as executive dysfunction is very common in this population [87].

ADL training also had a small to medium positive effect on physical endurance. After 3 months, participants who received ADL training were able to walk $31.2 \mathrm{~m}$ further in $6 \mathrm{~min}$ than participants who did not receive ADL training, which was considered clinically relevant $[88,89]$. This finding is encouraging, as performance on the 6MWT has been associated with strength, speed, balance, mood, and health factors in elderly individuals [90]. In addition, the 6MWT has been suggested to be reflective of the requirements of ADL performance [91], as walking difficulties can limit activity and participation in everyday life. No other physical 
improvements were observed following the ADL training, which contradicts a large study who report improved mobility, gait, and balance [24]. This could be due to the comparatively smaller sample size (87 vs. 486) and lower average MMSE score (12.3 vs. 20.4) in our study [24]. However, other studies did not find benefits of ADL training on physical performance $[26,29]$, which may suggest that the focus of the ADL intervention on training specific functional activities may not necessarily translate to improvements in physical functions.

Besides EF and physical endurance, ADL training resulted in reduced depressive symptoms among men, but not among women. Although this gender difference has not been described previously, positive effects of ADL training on depressive symptoms have been found in previous studies $[28,29]$. One explanation for these positive effects might be the specific focus of the ADL training on improving independence and participation in everyday activities, as giving participants more autonomy and control, and performing meaningful activities is found to benefit well-being [92]. Men and women experience depression for different reasons [93]. We hypothesize that the demonstrated gender differences in the effect of ADL training on depression may, to some degree, be the result of general gender differences in personal factors such as self-efficacy, preferred physical activity approaches, goals, and values [94]. The large ES found of the ADL intervention on depressive symptoms among men should be interpreted with caution, as small samples can produce inflated ES estimates [95].

With regard to the exercise intervention, we found that a multicomponent exercise training can benefit grip strength only of residents with mild-to-moderate cognitive impairment (MMSE score 15-23). This finding is supported by studies describing the relationship between poor cognition and impaired strength [96-98]. However, considering the limited power of the stratified analyses, we cannot conclude that exercise is not effective for residents with moderately severe-to-severe cognitive impairment (MMSE score 2-14). There is evidence that grip strength can be improved in $\mathrm{NH}$ residents with moderate-to-severe dementia [99]. Maintaining grip strength is important as it is an indicator of upper body strength [96] and is considered one of the most important muscle groups for performing ADLs [100]. We did not find any benefits of our multicomponent exercise intervention on other physical functions, cognitive functions, mood, or behavior. These results contradict recent meta-analyses, where multicomponent exercise improved physical functions [32-37] and cognitive functions [12, 40], and reduced depression and agitation [1, 32]. The lack of effect of exercise could be the result of the moderate treatment adherence (55\%) compared to similar studies $(>75 \%)[48,51,101]$. In addition, the limited amount of intensity reports suggests that participants trained at a lower than intended intensity (i.e., mainly 2 sets of repetitions instead of the intended 3, and a shorter than intended walking distance). High intensity and good adherence are essential for positive outcomes [43]. Since there were some deviations from the intended protocol, firm conclusions cannot be made regarding the effectiveness of our exercise intervention. However, we can conclude that an exercise intervention performed at a lower than intended intensity is not effective in improving outcomes of $\mathrm{NH}$ residents with dementia. Even so, our results are clinically relevant, as they show the complications of performing such an exercise intervention among $\mathrm{NH}$ residents with moderate-tosevere dementia.

We found some additional benefits of a combined exercise and ADL training over an ADL only, exercise only, and no physical activity intervention. Specifically, after 6 months, the combined intervention group showed improvements in functional mobility compared to the "ADL only" group, improvements in depressive symptoms and apathy compared to the "exercise only" group, and improvements in physical endurance compared to the no physical activity group. We hypothesize that the ADL intervention was the driving force for positive outcomes of the combined intervention. 
There are several possible explanations for the superiority of the ADL intervention over the exercise intervention. First, the higher adherence to the ADL training compared to the exercise intervention ( 68 vs. 55\%) in combination with the higher intensity of the ADL training ( 7 days per week in all care routines throughout the day vs. 3 times per week for 30-45 min). We cannot conclude which aspect of ADL training contributed to the positive effects on outcomes: the motivation from the staff, the cues, the physical activity, the practice itself, the one-on-one attention, or a combination of these factors. However, considering the limited effect of the exercise intervention, it is important to mention that the effects of ADL training may not be solely contributable to increased physical activity. The effects may also be due to the personal guidance and repeated practice of basic skills, in combination with promoting independence and psychological well-being. It is possible that more improvement would have occurred in the exercise group with individual treatment. However, this is difficult to realize in an NH setting.

\section{Strengths and Limitations}

Strengths of this study include the pragmatic nature of the interventions, which could be implemented in the NH setting. Second, the randomized design allowed for assessment of similar groups, which is a frequently reported limitation in similar studies [102]. Third, the majority of previous studies measuring cognitive functions were limited to measures of global cognition (MMSE). We measured a broader range of cognitive functions [32, 37, 39] and, besides the MMSE, included the SIB-S as a measure of global cognition, which is more sensitive to change than the MMSE in patients with moderate-to-severe dementia [61].

Some limitations should be considered. First, statistical inference is limited by the small sample size. As a result, we did not adjust our analyses for potential confounding factors, such as comorbidities, as this can lead to type 1 error when there is a small sample size [103]. However, the benefit of randomization is that it generates comparable groups with respect to variables that could influence the outcome, thereby minimizing confounding [104]. Second, the intensity of the exercise intervention was not consistently reported. Future research should pay extra attention to carefully reporting the exact intensity of the exercises, including number of sets, repetitions, and weight extensions for a strength training, and distance walked in meters for a walking training.

\section{Conclusion}

We conclude that ADL training is a feasible intervention that is effective in improving EF, physical endurance, and depressed mood of $\mathrm{NH}$ residents with moderately severe dementia. ADL training can easily be applied by nursing staff and enables continuous stimulation of movement throughout the day. No firm conclusions can be made regarding the effectiveness of our exercise intervention due to the moderate treatment adherence and limited number of intensity reports. We expect that a higher frequency or intensity of exercise training, as well as an individualized approach, may be necessary to stimulate changes. A combined exercise and ADL training appears to have some additional benefits over ADL, exercise, and no physical activity stimulation. More studies with larger samples are needed before firm conclusions can be drawn regarding the effectiveness of ADL training, exercise training, and a combined exercise and ADL training. However, our results highlight the important role which nursing staff play in preventing further disability through stimulating independence. 
Henskens et al.: Physical Activity in Nursing Home Residents with Dementia

\section{Statement of Ethics}

The Medical Ethics Review Committee of VU University Medical Center Amsterdam (2015.446) concluded that this study was not subject to the Medical Research Involving Human Subjects Act (WMO). Approval was granted by the scientific and Ethical Review Board (VCWE) of the Faculty of Behavior and Movement Sciences of VU University Amsterdam (VCWE-2015-183R1). Written informed consent was obtained from the legal representatives of the participants.

\section{Disclosure Statement}

This study is funded by Stichting tanteLouise, who contributed to the conception and design of the study, helped with data acquisition, and critically revised the manuscript. The study funders had no role in the analysis and interpretation of the data. The authors declare that they have no competing interests.

\section{Author Contributions}

M.H. conducted the study, supervised data collection, performed the data analysis and interpretation and writing of the manuscript. I.M.N. designed and conducted the study, supervised data collection, contributed to the data analysis and writing of the manuscript. M.C.A.E. advised in designing and conducting the study, contributed to data collection, and critically revised the manuscript. E.J.A.S. advised in designing the study, contributed to interpretation of data, and critically revised the manuscript. All authors read and approved the final manuscript.

\section{References}

1 Thuné-Boyle ICV, Iliffe S, Cerga-Pashoja A, Lowery D, Warner J: The effect of exercise on behavioral and psychological symptoms of dementia: towards a research agenda. Int Psychogeriatr 2012;24:1046-1057.

2 Eggermont LHP, Scherder EJA: Physical activity and behaviour in dementia: a review of the literature and implications for psychosocial intervention in primary care. Dementia 2006;5:411-428.

3 Scherder EJA, Bogen T, Eggermont LHP, Hamers JPH, Swaab DF: The more physical inactivity, the more agitation in dementia. Int Psychogeriatr 2010;22:1203-1208.

4 Archer T, Kostrzewa RM, Beninger RJ, Palomo T: Staging neurodegenerative disorders: structural, regional, biomarker, and functional progressions. Neurotox Res 2011;19:211-234.

5 Royall DR, Lauterbach EC, Cummings JL, Reeve A, Rummans TA, Kaufer DI, LaFrance WC Jr, Coffey CE: Executive control function: a review of its promise and challenges for clinical research. A report from the Committee on Research of the American Neuropsychiatric Association. J Neuropsychiatry Clin Neurosci 2002;14:377405.

6 Pettersson AF, Olsson E, Wahlund L-O: Motor function in subjects with mild cognitive impairment and early Alzheimer's disease. Dement Geriatr Cogn Disord 2005;19:299-304.

7 Zuidema SU, Derksen E, Verhey FRJ, Koopmans RTCM: Prevalence of neuropsychiatric symptoms in a large sample of Dutch nursing home patients with dementia. Int J Geriatr Psychiatry 2007;22:632-638.

8 Overshott R, Burns A: Treatment of dementia. J Neurol Neurosurg Psychiatry 2005;76:53-59.

9 Fleiner T, Leucht S, Förstl H, Zijlstra W, Haussermann P: Effects of short-term exercise interventions on behavioral and psychological symptoms in patients with dementia: a systematic review. J Alzheimers Dis 2017;55: 1583-1594.

10 Royall DR, Lauterbach EC, Kaufer D, Malloy P, Coburn KL, Black KJ: The cognitive correlates of functional status: a review from the Committee on Research of the American Neuropsychiatric Association. J Neuropsychiatry Clin Neurosci 2007;19:249-265.

11 Wetzels RB, Zuidema SU, de Jonghe JFM, Verhey FRJ, Koopmans RTCM: Determinants of quality of life in nursing home residents with dementia. Dement Geriatr Cogn Disord 2010;29:189-197.

12 Groot C, Hooghiemstra AM, Raijmakers PGHM, van Berckel BNM, Scheltens P, Scherder EJA, Van der Flier WM, Ossenkoppele R: The effect of physical activity on cognitive function in patients with dementia: a meta-analysis of randomized control trials. Ageing Res Rev 2016;25:13-23.

13 Colcombe S, Kramer AF: Fitness effects on the cognitive function of older adults: a meta-analytic study. Psychol Sci 2003;14:125-130.

14 Sofi F, Valecchi D, Bacci D, Abbate R, Gensini GF, Casini A, Macchi C: Physical activity and risk of cognitive decline: a meta-analysis of prospective studies. J Intern Med 2011;269:107-117. 
Henskens et al.: Physical Activity in Nursing Home Residents with Dementia

15 Scherder EJA, Van Paasschen J, Deijen J-BS, Van Der Knokke S, Orlebeke JFK, Burgers I, Devriese P-P, Swaab DF, Sergeant JA: Physical activity and executive functions in the elderly with mild cognitive impairment. Aging Ment Health 2005;9:272-280.

16 Hill K, Smith R, Fearn M, Rydberg M, Oliphant R: Physical and psychological outcomes of a supported physical activity program for older carers. J Aging Phys Act 2007;15:257-271.

17 Blake H, Mo P, Malik S, Thomas S: How effective are physical activity interventions for alleviating depressive symptoms in older people? A systematic review. Clin Rehabil 2009;23:873-887.

18 Pitkälä K, Savikko N, Poysti M, Strandberg T, Laakkonen M-L: Efficacy of physical exercise intervention on mobility and physical functioning in older people with dementia: a systematic review. Exp Gerontol 2013;48: 85-93.

19 Colcombe SJ, Erickson KI, Scalf PE, Kim JS, Prakash R, McAuley E, Elavsky S, Marquez DX, Hu L, Kramer AF: Aerobic exercise training increases brain volume in aging humans. J Gerontol A Biol Sci Med Sci 2006;61: 1166-1170.

20 Fjell AM, McEvoy L, Holland D, Dale AM, Walhovd KB, Initiative AsDN: What is normal in normal aging? Effects of aging, amyloid and Alzheimer's disease on the cerebral cortex and the hippocampus. Prog Neurobiol 2014; 117:20-40.

21 Forbes D, Forbes SC, Blake CM, Thiessen EJ, Forbes S: Exercise programs for people with dementia. Cochrane Database Syst Rev 2015;4:CD006489.

22 Giannakopoulos P, Hof PR, Giannakopoulos A-S, Buée-Scherrer V, Surini M, Delacourte A, Bouras C: Dementia in the oldest-old: quantitative analysis of 12 cases from a psychiatric hospital. Dement Geriatr Cogn Disord 1994;5:348-356.

23 Hooghiemstra AM, Eggermont LH, Scheltens P, van der Flier WM, Scherder EJA: Exercise and early-onset Alzheimer's disease: theoretical considerations. Dement Geriatr Cogn Disord Extra 2012;2:132-145.

24 Resnick B, Gruber-Baldini AL, Zimmerman S, Galik E, Pretzer-Aboff I, Russ K, Hebel JR: Nursing home resident outcomes from the res-care intervention. J Am Geriatr Soc 2009;57:1156-1165.

25 Farina E, Fioravanti R, Chiavari L, Imbornone E, Alberoni M, Pomati S, Pinardi G, Pignatti R, Mariani C: Comparing two programs of cognitive training in Alzheimer's disease: a pilot study. Acta Neurol Scand 2002; 105:365-371.

26 Resnick B, Galik E, Boltz M: Function focused care approaches: literature review of progress and future possibilities. J Am Med Dir Assoc 2013;14:313-318.

27 Resnick B, Galik E, Gruber-Baldini AL, Zimmerman S: Implementing a restorative care philosophy of care in assisted living: pilot testing of Res-Care-AL. J Am Acad Nurse Pract 2009;21:123-133.

28 Morris JN, Fiatarone M, Kiely DK, Belleville-Taylor P, Murphy K, Littlehale S, Ooi WL, O'Neill E, Doyle N: Nursing rehabilitation and exercise strategies in the nursing home. J Gerontol A Biol Sci Med Sci 1999;54:M494-M500.

29 Galik EM, Resnick B, Gruber-Baldini A, Nahm E-S, Pearson K, Pretzer-Aboff I: Pilot testing of the restorative care intervention for the cognitively impaired. J Am Med Dir Assoc 2008;9:516-522.

30 Talley KMC, Wyman JF, Savik K, Kane RL, Mueller C, Zhao H: Restorative care's effect on activities of daily living dependency in long-stay nursing home residents. Gerontologist 2015;55:S88-S98.

31 Resnick B, Simpson M, Bercovitz A, Galik E, Gruber-Baldini A, Zimmerman S, Magaziner J: Pilot testing of the restorative care intervention: impact on residents. J Gerontol Nurs 2006;32:39-47.

32 Brett L, Traynor V, Stapley P: Effects of physical exercise on health and well-being of individuals living with a dementia in nursing homes: a systematic review. J Am Med Dir Assoc 2016;17:104-116.

33 Lee HS, Park SW, Park YJ: Effects of physical activity programs on the improvement of dementia symptom: a meta-analysis. Biomed Res Int 2016;2016.

34 Rolland Y, Pillard F, Klapouszczak A, Reynish E, Thomas D, Andrieu S, Rivière D, Vellas B: Exercise program for nursing home residents with Alzheimer's disease: a 1-year randomized, controlled trial. J Am Geriatr Soc 2007; 55:158-165.

35 Potter R, Ellard D, Rees K, Thorogood M: A systematic review of the effects of physical activity on physical functioning, quality of life and depression in older people with dementia. Int J Geriatr Psychiatry 2011;26: 1000-1011.

36 Blankevoort CG, Van Heuvelen MJG, Boersma F, Luning H, De Jong J, Scherder EJA: Review of effects of physical activity on strength, balance, mobility and ADL performance in elderly subjects with dementia. Dement Geriatr Cogn Disord 2010;30:392-402.

37 Sampaio A, Marques EA, Mota J, Carvalho J: Effects of a multicomponent exercise program in institutionalized elders with Alzheimer's disease. Dementia 2016;1471301216674558.

38 Hess NC, Dieberg G, McFarlane JR, Smart NA: The effect of exercise intervention on cognitive performance in persons at risk of, or with, dementia: a systematic review and meta-analysis. Healthy Aging Res 2014;3:1-10.

39 Farina N, Rusted J, Tabet N: The effect of exercise interventions on cognitive outcome in Alzheimer's disease: a systematic review. Int Psychogeriatr 2014;26:9-18.

40 Heyn P, Abreu BC, Ottenbacher KJ: The effects of exercise training on elderly persons with cognitive impairment and dementia: a meta-analysis. Arch Phys Med Rehabil 2004;85:1694-1704.

41 Verghese J, Wang C, Lipton RB, Holtzer R, Xue X: Quantitative gait dysfunction and risk of cognitive decline and dementia. J Neurol Neurosurg Psychiatry 2007;78:929-935. 
42 Bossers WJ, van der Woude LH, Boersma F, Hortobágyi T, Scherder EJA, van Heuvelen MJ: A 9-week aerobic and strength training program improves cognitive and motor function in patients with dementia: a randomized, controlled trial. Am J Geriatr Psychiatry 2015;23:1106-1116.

43 Öhman H, Savikko N, Strandberg TE, Pitkälä KH: Effect of physical exercise on cognitive performance in older adults with mild cognitive impairment or dementia: a systematic review. Dement Geriatr Cogn Disord 2014; 38:347-365.

44 Scherder EJA, Scherder R, Verburgh L, Königs M, Blom M, Kramer AF, Eggermont L: Executive functions of sedentary elderly may benefit from walking: a systematic review and meta-analysis. Am J Geriatr Psychiatry 2014;22:782-791.

45 Souto Barreto P, Cesari M, Denormandie P, Armaingaud D, Vellas B, Rolland Y: Exercise or social intervention for nursing home residents with dementia: a pilot randomized, controlled trial. J Am Geriatr Soc 2017;65:9.

46 Forbes D, Forbes S, Morgan DG, Markle-Reid M, Wood J, Culum I: Physical activity programs for persons with dementia. Cochrane Database Syst Rev 2008;3:CD006489.

47 Littbrand H, Stenvall M, Rosendahl E: Applicability and effects of physical exercise on physical and cognitive functions and activities of daily living among people with dementia: a systematic review. Am J Phys Med Rehabil 2011;90:495-518.

48 Forbes D, Thiessen EJ, Blake CM, Forbes SC, Forbes S: Exercise programs for people with dementia. Cochrane Database Syst Rev 2013;12:0.

49 Steinberg M, Leoutsakos JMS, Podewils LJ, Lyketsos C: Evaluation of a home-based exercise program in the treatment of Alzheimer's disease: the Maximizing Independence in Dementia (MIND) study. Int J Geriatr Psychiatry 2009;24:680-685.

50 Edwards N, Gardiner M, Ritchie DM, Baldwin K, Sands L: Effect of exercise on negative affect in residents in special care units with moderate to severe dementia. Alzheimer Dis Assoc Disord 2008;22:362-368.

51 Venturelli M, Scarsini R, Schena F: Six-month walking program changes cognitive and ADL performance in patients with Alzheimer. Am J Alzheimers Dis Other Demen 2011;26:381-388.

52 Henskens M, Nauta IM, Drost KT, Scherder EJA: The effects of movement stimulation on activities of daily living performance and quality of life in nursing home residents with dementia: a randomized controlled trial. Clin Interv Aging 2018;13:805.

53 Graessel E, Viegas R, Stemmer R, Küchly B, Kornhuber J, Donath C: The Erlangen Test of Activities of Daily Living: first results on reliability and validity of a short performance test to measure fundamental activities of daily living in dementia patients. Int Psychogeriatr 2009;21:103-112.

54 Huntleigh A: ARJO Guidebook for Architects and Planners: Elderly Care Facilities: ARJO Hospital Equipment. Malmö, ARJO, 2005.

55 Folstein MF, Folstein SE, McHugh PR: "Mini-mental state": a practical method for grading the cognitive state of patients for the clinician. J Psychiatr Res 1975;12:189-198.

56 Rikli RE, Jones CJ: The reliability and validity of a 6-minute walk test as a measure of physical endurance in older adults. J Aging Phys Act 1998;6:363-375.

57 Verhage F: Intelligentie en leeftijd: onderzoek bij Nederlanders van twaalf tot zevenenzeventig jaar. Assen, Van Gorcum, 1964.

58 Reisberg B, Ferris SH, de Leon MJ, Crook T: The Global Deterioration Scale for assessment of primary degenerative dementia. Am J Psychiatry 1982;139:1136-1139.

59 Centers for Medicare \& Medicaid Services: Revised Long-Term Care Facility Resident Assessment Instrument User's Manual Version 2.0. Baltimore, CMS, 2008.

60 Saxton J, Kastango KB, Hugonot-Diener L, Boller F, Verny M, Sarles CE, Girgis RR, Devouche E, Mecocci P, Pollock BG, DeKosky ST: Development of a short form of the Severe Impairment Battery. Am J Geriatric Psychiatry 2005;13:999-1005.

61 Bossers WJ, Van der Woude LH, Boersma F, Scherder EJA, van Heuvelen MJ: Recommended measures for the assessment of cognitive and physical performance in older patients with dementia: a systematic review. Dement Geriatr Cogn Disord 2012;2:589-609.

62 Brucki SMD, Rocha MSG: Category fluency test: effects of age, gender and education on total scores, clustering and switching in Brazilian Portuguese-speaking subjects. Braz J Med Biol Res 2004;37:1771-1777.

63 Snijders JT, Verhage F: Groninger intelligentie test. Lisse, Swets \& Zeitlinger, 1983.

64 Wechsler D: WMS-R: Wechsler Memory Scale - Revised. San Antonio, Psychological Corporation, 1987.

65 Dubois B, Slachevsky A, Litvan I, Pillon B: The FAB: a frontal assessment battery at bedside. Neurology 2000; 55:1621-1626.

66 Podsiadlo D, Richardson S: The timed "Up \& Go": a test of basic functional mobility for frail elderly persons. J Am Geriatr Soc 1991;39:142-148.

67 Hess RJ, Brach JS, Piva SR, Van Swearingen JM: Walking skill can be assessed in older adults: validity of the Figure-of-8 Walk Test. Phys Ther 2010;90:89-99.

68 Rossiter-Fornoff JE, Wolf SL, Wolfson LI, Buchner DM: A cross-sectional validation study of the FICSIT common data base static balance measures. J Gerontol A Biol Sci Med Sci 1995;50:M291-M297.

69 Bohannon RW: Sit-to-stand test for measuring performance of lower extremity muscles. Percept Mot Skills 1995;80:163-166.

70 Schaubert KL, Bohannon RW: Reliability and validity of three strength measures obtained from communitydwelling elderly persons. J Strength Cond Res 2005;19:717-720. 
Henskens et al.: Physical Activity in Nursing Home Residents with Dementia

71 Ries JD, Echternach JL, Nof L, Gagnon Blodgett M: Test-retest reliability and minimal detectable change scores for the timed "up \& go" test, the six-minute walk test, and gait speed in people with Alzheimer disease. Phys Ther 2009;89:569-579.

72 Blankevoort CG, Van Heuvelen MJG, Scherder EJA: Reliability of six physical performance tests in older people with dementia. Phys Ther 2013;93:69-78.

73 Schwartz S, Cohen ME, Herbison GJ, Shah A: Relationship between two measures of upper extremity strength: manual muscle test compared to hand-held myometry. Arch Phys Med Rehabil 1992;73:1063-1068.

74 Droës R: Dutch Translation of the Cornell-Scale for Depression in Dementia. Amsterdam, VU University, 1993.

75 Lueken U, Seidl U, Völker L, Schweiger E, Kruse A, Schröder J: Development of a short version of the Apathy Evaluation Scale specifically adapted for demented nursing home residents. Am J Geriatric Psychiatry 2007; 15:376-385.

76 Cohen-Mansfield J, Marx MS, Rosenthal AS: A description of agitation in a nursing home. J Gerontol 1989; 44:M77-M84.

77 Jonghe JFM, Kat MG: Factor structure and validity of the Dutch version of the Cohen-Mansfield Agitation Inventory (CMAI-D). J Am Geriatr Soc 1996;44:888-889.

78 Leontjevas R, Gerritsen DL, Vernooij-Dassen MJ, Smalbrugge M, Koopmans RT: Comparative validation of proxy-based Montgomery-Åsberg depression rating scale and Cornell scale for depression in dementia in nursing home residents with dementia. Am J Geriatr Psychiatry 2012;20:985-993.

79 Zuidema SU, Buursema AL, Gerritsen MGJM, Oosterwal KC, Smits MMM, Koopmans RTCM, de Jonghe JFM: Assessing neuropsychiatric symptoms in nursing home patients with dementia: reliability and reliable change index of the Neuropsychiatric Inventory and the Cohen-Mansfield Agitation Inventory. Int J Geriatr Psychiatry 2011;26:127-134.

80 Twisk JW: Applied Longitudinal Data Analysis for Epidemiology: A Practical Guide. Cambridge, Cambridge University Press, 2003.

81 Twisk J, de Boer M, de Vente W, Heymans M: Multiple imputation of missing values was not necessary before performing a longitudinal mixed-model analysis. J Clin Epidemiol 2013;66:1022-1028.

82 Cohen J: Statistical Power Analysis for the Behavioral Sciences, ed 2. Hillsdale, Erlbaum, 1988.

83 Helmer C, Pérès K, Letenneur L, Guttiérez-Robledo LM, Ramaroson H, Barberger-Gateau P, Fabrigoule C, Orgogozo J-M, Dartigues J-F: Dementia in subjects aged 75 years or over within the PAQUID cohort: prevalence and burden by severity. Dement Geriatr Cogn Disord 2006;22:87-94.

84 Nebes RD, Martin DC, Horn LC: Sparing of semantic memory in Alzheimer's disease. J Abnorm Psychol 1984; 93:321.

85 Martyr A, Clare L: Executive function and activities of daily living in Alzheimer's disease: a correlational metaanalysis. Dement Geriatr Cogn Disord 2012;33:189-203.

86 Shao Z, Janse E, Visser K, Meyer AS: What do verbal fluency tasks measure? Predictors of verbal fluency performance in older adults. Front Psychol 2014;5:772.

87 Workman RH Jr, McCullough LB, Molinari V, Kunik ME, Orengo C, Khalsa DK, Rezabek P: Clinical and ethical implications of impaired executive control functions for patient autonomy. Psychiatr Serv 2000;51:359-363.

88 McDonald CM, Henricson EK, Abresch RT, Florence J, Eagle M, Gappmaier E, Glanzman AM, Spiegel R, Barth J, Elfring G, Reha A, Peltz SW: The 6-min walk test and other clinical endpoints in Duchenne muscular dystrophy: reliability, concurrent validity, and minimal clinically important differences from a multicenter study. Muscle Nerve 2013;48:357-368.

89 Bohannon RW, Crouch R: Minimal clinically important difference for change in 6-minute walk test distance of adults with pathology: a systematic review. J Eval Clin Pract 2017;23:377-381.

90 Lord S, Menz H: Performance in the six-minute walk test depends on multiple physiological, psychological and health factors in older people. Arch Phys Med Rehabil 2002;83:907-911.

91 Solway S, Brooks D, Lacasse Y, Thomas S: A qualitative systematic overview of the measurement properties of functional walk tests used in the cardiorespiratory domain. Chest 2001;119:256-270.

92 Rijnaard M, van Hoof J, Janssen BM, Verbeek H, Pocornie W, Eijkelenboom A, Beerens HC, Molony SL, Wouters EJM: The factors influencing the sense of home in nursing homes: a systematic review from the perspective of residents. J Aging Res 2016;2016:6143645.

93 Fuhrer R, Dufouil C, Dartigues JF: Exploring sex differences in the relationship between depressive symptoms and dementia incidence: prospective results from the PAQUID Study. J Am Geriatr Soc 2003;51:1055-1063.

94 Ekkekakis P, Hall EE, Petruzzello SJ: Variation and homogeneity in affective responses to physical activity of varying intensities: an alternative perspective on dose-response based on evolutionary considerations. J Sports Sci 2005;23:477-500.

95 Button KS, Ioannidis JP, Mokrysz C, Nosek BA, Flint J, Robinson ES, Munafò MR: Power failure: why small sample size undermines the reliability of neuroscience. Nat Rev Neurosci 2013;14:365.

96 Rogers SD, Jarrott SE: Cognitive impairment and effects on upper body strength of adults with dementia. J Aging Phys Act 2008;16:61-68.

97 Raji MA, Kuo YF, Snih SA, Markides KS, Peek MK, Ottenbacher KJ: Cognitive status, muscle strength, and subsequent disability in older Mexican Americans. J Am Geriatr Soc 2005;53:1462-1468.

98 Thomas VS: Excess functional disability among demented subjects? Dement Geriatr Cogn Disord 2001;12: 206-210. 
99 Henwood T, Neville C, Baguley C, Clifton K, Beattie E: Physical and functional implications of aquatic exercise for nursing home residents with dementia. Geriatr Nurs 2015;36:35-39.

100 Hunter GR, Treuth MS, Weinsier RL, Kekes-Szabo T, Kell SH, Roth DL, Nicholson C: The effects of strength conditioning on older women's ability to perform daily tasks. J Am Geriatr Soc 1995;43:756-760.

101 Santana-Sosa E, Barriopedro MI, López-Mojares LM, Pérez M, Lucia A: Exercise training is beneficial for Alzheimer's patients. Int J Sports Med 2008;29:845-850.

102 Ojagbemi A, Akin-Ojagbemi N: Exercise and quality of life in dementia: a systematic review and meta-analysis of randomized controlled trials. J Appl Gerontol 2017;0733464817693374.

103 Kahan BC, Jairath V, Doré CJ, Morris TP: The risks and rewards of covariate adjustment in randomized trials: an assessment of 12 outcomes from 8 studies. Trials 2014;15:139.

104 Suresh KP: An overview of randomization techniques: an unbiased assessment of outcome in clinical research. J Hum Reprod Sci 2011;4:8-11. 